\title{
Article \\ Unusual Temperature Evolution of Quasiparticle Band Dispersion in Electron-Doped FeSe Films
}

\author{
Kosuke Nakayama ${ }^{1,2, *(\mathbb{D})}$, Koshin Shigekawa ${ }^{1}$, Katsuaki Sugawara ${ }^{1,2,3,4}$, Takashi Takahashi ${ }^{1,3,4}$ \\ and Takafumi Sato $1,3,4$ \\ 1 Department of Physics, Tohoku University, Sendai 980-8578, Japan; \\ k.shigekawa@arpes.phys.tohoku.ac.jp (K.S.); k.sugawara@arpes.phys.tohoku.ac.jp (K.S.); \\ t.takahashi@arpes.phys.tohoku.ac.jp (T.T.); t-sato@arpes.phys.tohoku.ac.jp (T.S.) \\ 2 Precursory Research for Embryonic Science and Technology (PRESTO), \\ Japan Science and Technology Agency (JST), Tokyo 102-0076, Japan \\ 3 WPI Research Center, Advanced Institute for Materials Research, Tohoku University, Sendai 980-8577, Japan \\ 4 Center for Spintronics Research Network, Tohoku University, Sendai 980-8577, Japan \\ * Correspondence: k.nakayama@arpes.phys.tohoku.ac.jp
}

check for

updates

Citation: Nakayama, K.; Shigekawa, K.; Sugawara, K.; Takahashi, T.; Sato, T. Unusual Temperature Evolution of Quasiparticle Band Dispersion in Electron-Doped FeSe Films. Symmetry 2021, 13, 155. https://doi.org/ 10.3390/sym13020155

Received: 28 December 2020

Accepted: 18 January 2021

Published: 20 January 2021

Publisher's Note: MDPI stays neutral with regard to jurisdictional claims in published maps and institutional affiliations.

Copyright: (c) 2021 by the authors. Licensee MDPI, Basel, Switzerland. This article is an open access article distributed under the terms and conditions of the Creative Commons Attribution (CC BY) license (https:/ / creativecommons.org/licenses/by/ $4.0 /)$.

\begin{abstract}
The discovery of high-temperature (high- $T_{\mathrm{c}}$ ) superconductivity in one-monolayer FeSe on $\mathrm{SrTiO}_{3}$ has attracted tremendous attention. Subsequent studies suggested the importance of cooperation between intra-FeSe-layer and interfacial interactions to enhance $T_{\mathrm{c}}$. However, the nature of intra-FeSe-layer interactions, which would play a primary role in determining the pairing symmetry, remains unclear. Here we have performed high-resolution angle-resolved photoemission spectroscopy of one-monolayer and alkaline-metal-deposited multilayer FeSe films on $\mathrm{SrTiO}_{3}$, and determined the evolution of quasiparticle band dispersion across $T_{\mathrm{c}}$. We found that the band dispersion in the superconducting state deviates from the Bogoliubov-quasiparticle dispersion expected from the normal-state band dispersion with a constant gap size. This suggests highly anisotropic pairing originating from small momentum transfer and/or mass renormalization due to electron-boson coupling. This band anomaly is interpreted in terms of the electronic interactions within the FeSe layers that may be related to the high- $T_{\mathrm{C}}$ superconductivity in electron-doped FeSe.
\end{abstract}

Keywords: iron-based superconductors; thin films; ARPES; electronic structure

\section{Introduction}

Iron selenide (FeSe) is structurally the simplest iron-based superconductor with the superconducting-transition temperature $\left(T_{\mathrm{c}}\right)$ of $\sim 9 \mathrm{~K}$ [1]. Intriguingly, one-monolayer (1 ML) film of FeSe grown on $\mathrm{SrTiO}_{3}$ substrate exhibits exceptionally high $T_{\mathrm{c}}$ [2]. The $T_{\mathrm{c}}$ value reported by transport measurements reaches $40 \mathrm{~K}[2,3]$, which is about five times higher than the bulk counterpart. In addition, Cooper pairing at a higher temperature of $65 \mathrm{~K}$, which exceeds the highest $T_{\mathrm{c}}(56 \mathrm{~K})$ ever achieved in iron-based superconductors, has been suggested from a gap-closing temperature by angle-resolved photoemission spectroscopy (ARPES) [4-7] and Meissner effect by mutual conductance measurements [8]. These observations triggered fierce debates on the origin of the $T_{\mathrm{c}}$ enhancement in $1 \mathrm{ML}-$ FeSe film. One key ingredient is a novel cross-interface electron-phonon coupling. The strong coupling between electrons in the FeSe layer and optical phonons of $\mathrm{SrTiO}_{3}$ has been verified via the observation of replica bands by ARPES and theoretically proposed to enhance $T_{\mathrm{c}}$ in most of possible pairing symmetries $[9,10]$. Later, the close link between strong electron-phonon coupling and $T_{\mathrm{c}}$ enhancement has been supported experimentally, e.g., by isotope effects $[11,12]$. With these findings as a guiding principle, the search for high $T_{\mathcal{C}}$ in atomically thin films of other iron-based superconductors interfaced with $\mathrm{SrTiO}_{3}$ [13-15] has been accelerated. Another key ingredient for $T_{\mathrm{c}}$ enhancement is a charge transfer from $\mathrm{SrTiO}_{3}$. Heavy electron doping to the FeSe layer leads to unique electronic structure 
consisting only of electron-like Fermi surfaces $[4,5,16]$, in contrast to the semimetallic nature of bulk FeSe [17,18]. The electron doping is essential for the high- $T_{\mathrm{c}}$ superconductivity, as established by the observation of high $T_{\mathrm{c}}$ above $40 \mathrm{~K}$ even in multilayer and bulk FeSe by doping electron carriers [19-21]. Therefore, there is accumulated evidence that the FeSe layer has the capability of inducing $40-\mathrm{K}$ superconductivity through electron doping and the interfacial electron-phonon coupling will assist further $T_{\mathrm{c}}$ or pairing enhancement. However, little is known about why electron doping leads to the high $T_{\mathrm{c}}$ 's above $40 \mathrm{~K}$. In particular, interactions within the FeSe layer, which would primarily determine the pairing symmetry, remain unclear.

In this study, we performed a comparative ARPES experiment on the surface of $1 \mathrm{ML}$ and Cs-deposited $20 \mathrm{ML}-\mathrm{FeSe}$ films on $\mathrm{SrTiO}_{3}$, where the interfacial effects were present and absent, respectively. We demonstrated anomaly in the quasiparticle-band dispersions in the superconducting state, which is not expected from the Bogoliubov-quasiparticle (BQP) dispersion induced by a simple s-wave-gap opening. We discuss implications of our observation in relation to intra-FeSe-layer interactions.

\section{Materials and Methods}

The molecular beam epitaxy method was used to obtain 1 ML- and 20 ML-FeSe films; the films were grown on a $\mathrm{TiO}_{2}$-terminated $\mathrm{Nb}(0.05 \mathrm{wt} \%)-\mathrm{SrTiO}_{3}$ substrate (SHINKOSHA) by simultaneously evaporating Fe and Se atoms while keeping a substrate temperature at $430{ }^{\circ} \mathrm{C}$ with a deposition rate of $0.01 \mathrm{ML} / \mathrm{s}$ [19]. Electron doping to $20 \mathrm{ML}-\mathrm{FeSe}$ was realized by evaporating $\mathrm{Cs}$ atoms onto the film surface at room temperature using a Cs dispenser (SAES Getters) [22]. After the growth, the film was transferred to the ARPES-measurement chamber without exposure to air. ARPES measurements were performed with a SES2002 spectrometer (Scienta Omicron) with the He-I $\alpha$ resonance line $(h v=21.218 \mathrm{eV})$ at Tohoku University. The film was kept under an ultrahigh vacuum of $5 \times 10^{-11}$ Torr during the ARPES measurement, and no remarkable surface degradation was observed for a typical measurement time of 1 day. The energy and angular resolutions were set to be $7-12 \mathrm{meV}$ and $0.2^{\circ}$, respectively. A gold film which made electrical contact with the film was referenced to calibrate the Fermi level $\left(E_{\mathrm{F}}\right)$.

\section{Results}

First, we present the electronic structure of Cs-deposited $20 \mathrm{ML}-F e S e$ film measured at $T=50 \mathrm{~K}$. As shown in Figure $1 \mathrm{a}, \mathrm{b}$, there was a circular-shaped large Fermi surface at the Brillouin-zone corner (M point) which originated from $E_{\mathrm{F}}$ crossing of an electron band with the bottom of the dispersion around $50 \mathrm{meV}$ below $E_{\mathrm{F}}$. The top of a hole-like band around the zone center ( $\Gamma$ point) was about $50 \mathrm{meV}$ below $E_{\mathrm{F}}$, resulting in the absence of a hole-like Fermi surface in contrast to the as-grown multilayer FeSe film [4,5] or bulk FeSe [17,18]. These observations confirmed a successful electron doping by Cs deposition onto the FeSe surface. The electron carrier concentration $\left(n_{\mathrm{e}}\right)$ calculated from the Fermi-surface volume was $\sim 0.11$ electrons $/ \mathrm{Fe}$, which corresponds to the optimal doping level with $T_{\mathrm{c}}$ value of $\sim 40 \mathrm{~K}$ [22]. To investigate how the band structure changes by the superconducting transition, we performed high-resolution measurements across $T_{\mathrm{c}}$ (50 and $13 \mathrm{~K}$ ) along a momentum $(k)$ cut $\mathrm{A}$ indicated by a blue line in Figure 1a. The results displayed in Figure $1 c$,d show that while the electron band above $T_{\mathrm{c}}$ crossed $E_{\mathrm{F}}$ at the Fermi wave vector $\left(k_{\mathrm{F}}\right)$ of $\sim 0.17 \pi / a$, the band dispersion below $T_{\mathrm{C}}$ had a local maximum below $E_{\mathrm{F}}$ so as not to cross $E_{\mathrm{F}}$ due to a superconducting-gap opening. It is noted that the $k$ location of the electron-band top below $T_{\mathrm{C}}$ coincided with the $k_{\mathrm{F}}$ point above $T_{\mathrm{c}}$, consistent with the Cooper-pairing origin of the observed gap. The superconducting-gap opening is also clearly seen in energy distribution curves (EDCs) in Figure 1e, in which the peak position at $k_{\mathrm{F}}\left(k_{3}\right.$ defined in Figure $\left.1 \mathrm{c}, \mathrm{d}\right)$ was shifted from $E_{\mathrm{F}}$ to a high binding energy by $\sim 10 \mathrm{meV}$ with decreasing the temperature to form a superconducting gap. Since the nodeless $s$ wave superconductivity is realized in electron-doped multilayer FeSe $[19,20]$, one can see the superconducting-gap opening below $T_{\mathrm{c}}$ irrespective of the $k$ cut, e.g., along the $k$ cut 
crossing the M point (cut B), as shown in Figure 1f-h, where essentially the same behavior with cut A was recognized.
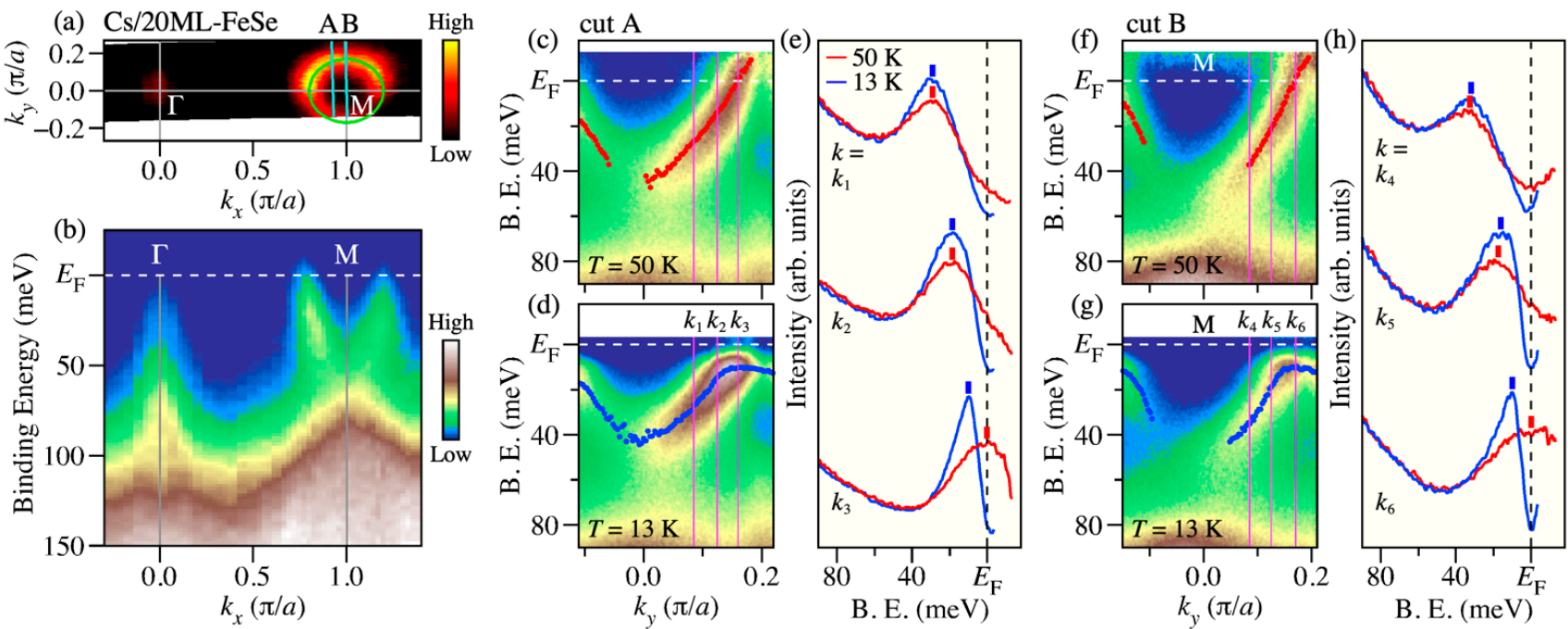

Figure 1. (a) ARPES intensity map at $E_{\mathrm{F}}$ as a function of two-dimensional wave vector for Cs-deposited 20 monolayer (ML)-FeSe film obtained at $T=50 \mathrm{~K}$ with $h v=21.218 \mathrm{eV}$. Intensity at $E_{\mathrm{F}}$ was obtained by integrating the spectral intensity within $\pm 10 \mathrm{meV}$ of $E_{\mathrm{F}}$. Green circle is a guide for the eyes to trace the Fermi surface. (b) Plot of ARPES intensity along the $\Gamma \mathrm{M}$ cut at $50 \mathrm{~K}$ as a function of binding energy and wave vector. (c,d) Near- $E_{\mathrm{F}}$ ARPES intensity along cut $\mathrm{A}$ in (a) at $T=50$ and $13 \mathrm{~K}$, respectively, divided by the Fermi-Dirac distribution (FD) function at each temperature convoluted with the resolution function. Intensity above $E_{\mathrm{F}}$ is displayed up to $3 k_{\mathrm{B}} T$. Red and blue circles in (c,d), respectively, are the band dispersion determined by fitting the energy distribution curves (EDCs) with Bardeen-Cooper-Schrieffer (BCS) spectral function [23]. (e) Comparison of EDCs between $T=50 \mathrm{~K}$ (red) and $13 \mathrm{~K}$ (blue) taken at representative $k_{y}$ points [ $k_{1}, k_{2}$, and $k_{3}$ indicated by magenta lines in (c,d)]. Red and blue dots indicate the local maxima corresponding to the peak position. $(\mathbf{f}-\mathbf{h})$ Same as (c-f) but obtained along cut B in (a).

An important finding manifests itself when we compare the band dispersions of the normal and superconducting states. Figure 2a displays a direct comparison of the experimental band dispersions extracted from the peak position of EDCs in cut A. As mentioned above, the band dispersion below $T_{\mathrm{C}}$ exhibited an opening of the superconducting gap and resultant bending-back behavior with the top of the dispersion at $k_{\mathrm{F}}$. Such a characteristic band dispersion below $T_{\mathrm{C}}$ was qualitatively consistent with the dispersion relation of BQPs in the Bardeen-Cooper-Schrieffer (BCS) theory, where BQP dispersion $\left(E_{k}\right)$ is expressed as $E_{k}=\sqrt{\varepsilon_{k}^{2}+|\Delta|^{2}}\left(\varepsilon_{k}\right.$ and $\Delta$ are the normal-state band dispersion and the superconducting-gap size, respectively) [24]. For a quantitative comparison, we determined $\varepsilon_{k}$ by performing a polynomial fitting to the ARPES data above $T_{\mathrm{c}}$ (magenta curve) and simulated $E_{k}$ by assuming a $k$-independent superconducting-gap size of $10 \mathrm{meV}$ (light blue curve). Intriguingly, the band dispersion below $T_{\mathrm{c}}$ shows a clear deviation from the simulated $\mathrm{BQP}$ dispersion $E_{k}$; specifically, although the simulation predicted a finite downward energy shift of BQP dispersion compared with $\varepsilon_{k}$ even in the $k$ region far away from $k_{\mathrm{F}}$ (at least down to $k_{y}=0.05 \pi / a$ ) because of a large $\Delta$ value with respect to the shallow electron-band bottom, the experimental dispersion below $T_{\mathrm{c}}$ became nearly identical to $\varepsilon_{k}$ as soon as it moved away from $k_{\mathrm{F}}$. Almost temperature-insensitive band position away from $k_{\mathrm{F}}$ was also clearly visible in the comparison of raw EDCs in Figure 1e (see EDCs at $k_{1}$ and $k_{2}$ ). The same behavior was observed at different momentum, e.g., we found that the band dispersion measured below $T_{\mathrm{C}}$ along cut $\mathrm{B}$ deviated from the simulated $\mathrm{BQP}$ dispersion over a wide $k$ region (see Figure $2 \mathrm{~b}$; also see a comparison of EDCs in Figure $1 \mathrm{~h}$ ). 

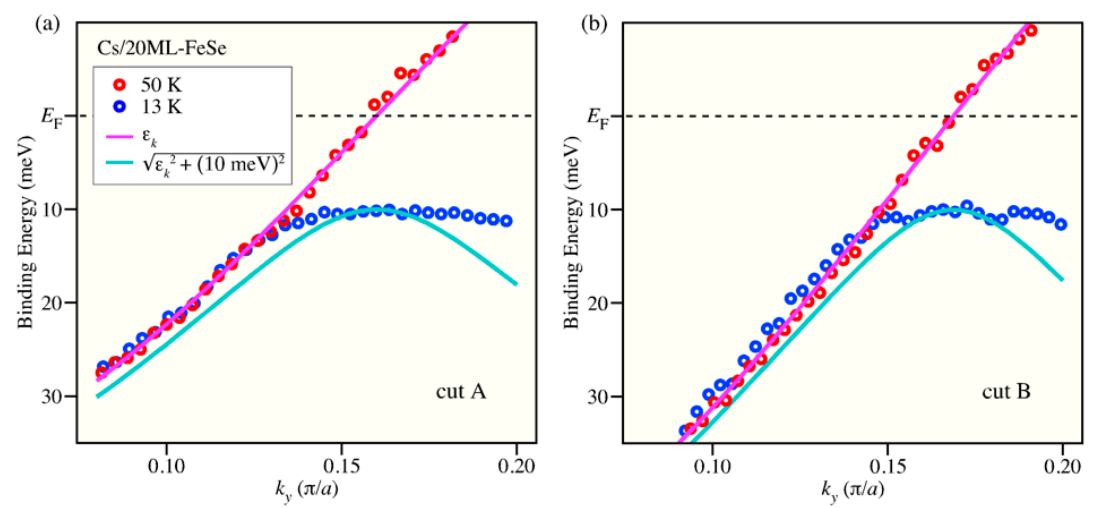

Figure 2. (a) Comparison of the near- $E_{\mathrm{F}}$ band dispersions in Cs-deposited $20 \mathrm{ML}-\mathrm{FeSe}$ at $\mathrm{T}=50 \mathrm{~K}$ (red circles) and $13 \mathrm{~K}$ (blue circles) along cut A in Figure 1a. Magenta curve is the normal-state band dispersion $\varepsilon_{k}$ extracted from a polynomial fitting to the red circles. Light blue curve is the calculated Bogoliubov-quasiparticle (BQP) dispersion based on the BCS formula $E_{k}=\sqrt{\varepsilon_{k}^{2}+|\Delta|^{2}}$ with a constant $\Delta$ of $10 \mathrm{meV}$. (b) Same as (a) but for cut B in Figure 1a.

To clarify whether the energy difference between the experimental and simulated BQP dispersions below $T_{\mathrm{C}}$ was an essential ingredient of electron-doped high- $T_{\mathrm{C}}$ FeSe films, we investigated the band-structure evolution in $1 \mathrm{ML}-\mathrm{FeSe}$ (Figure 3). For this purpose, we performed high-resolution measurements on slightly underdoped $1 \mathrm{ML}-\mathrm{FeSe}\left(n_{\mathrm{e}}=0.09\right)$ with $T_{\mathrm{c}} \sim 40 \mathrm{~K}$ because a sharp spectral line shape compared with the heavily doped sample ( $T_{\mathrm{c}} \sim 65 \mathrm{~K} ; n_{\mathrm{e}} \sim 0.12$ ) [4] is suited for accurately determining the quasiparticle band dispersion. As is well known, $1 \mathrm{ML}$-FeSe has a large electron-like Fermi surface centered at the $\mathrm{M}$ point. The electron band which forms the Fermi surface showed an opening of the superconducting gap $(\Delta \sim 10 \mathrm{meV})$ below $T_{\mathrm{c}}$, as highlighted by the characteristic bendingback behavior with the minimum-gap locus at $k_{\mathrm{F}}$ (see Figure $3 \mathrm{~b}$ ). A direct comparison of the band dispersions above and below $T_{\mathrm{c}}$ in Figure $3 \mathrm{c}$ demonstrates that an energy shift due to the superconducting-gap opening was limited to the $k$ region around $k_{\mathrm{F}} \sim 0.16 \pi / a$ (compare red and blue circles), in sharp contrast to a clear downward shift of the simulated $\mathrm{BQP}$ dispersion for $k_{y} \leq 0.1 \pi / a$ (light blue curve). Similarly, the case of Cs-deposited $20 \mathrm{ML}-\mathrm{FeSe}$ suggested that the deviation of the experimental band dispersion from the simulated $\mathrm{BQP}$ dispersion below $T_{\mathrm{c}}$ is a common feature of electron-doped FeSe films irrespective of film thickness.
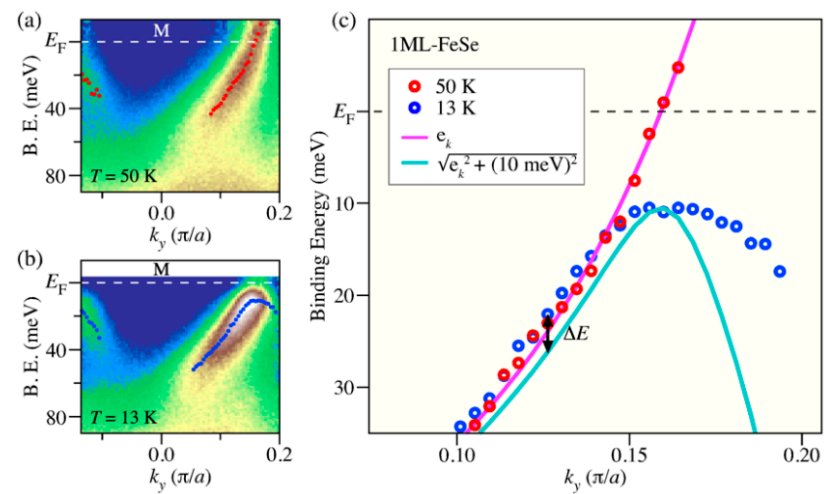

Figure 3. $(\mathbf{a}, \mathbf{b})$ ARPES intensity divided by the FD function measured along the $k$ cut crossing the M point in $1 \mathrm{ML}-\mathrm{FeSe}$ at $T=50 \mathrm{~K}$ and $13 \mathrm{~K}$, respectively. Red and blue circles show the band dispersion extracted from the peak position of the EDCs. (c) Comparison of the near- $E_{\mathrm{F}}$ band dispersions at $T=50 \mathrm{~K}$ (red circles) and $13 \mathrm{~K}$ (blue circles), together with $\varepsilon_{k}$ determined by polynomial fitting to the red circles (magenta curve) and the BQP dispersion $E_{k}=\sqrt{\varepsilon_{k}^{2}+|\Delta|^{2}}$ simulated with a constant $\Delta$ of $10 \mathrm{meV}$ (light blue curve). 


\section{Discussion and Conclusions}

Now we are going to discuss the origin of the observed anomaly in quasiparticle dispersion. To simulate $\mathrm{BQP}$ dispersion $E_{k}=\sqrt{\varepsilon_{k}^{2}+|\Delta|^{2}}$, we assumed that $\varepsilon_{k}$ is the same as the dispersion above $T_{\mathrm{c}}$ and $\Delta$ is $k$-independent. It would be natural to consider that one or both of these assumptions are incorrect, rather than thinking that the BQP picture was broken in the electron-doped FeSe. For simplicity, we consider in the following the two extreme cases that the deviation was induced by a change in either $\varepsilon_{k}$ or $\Delta$. First, to examine the $k$ dependence of $\Delta$ as the origin, we put the experimental band dispersions below and above $T_{\mathrm{c}}$ into $E_{k}$ and $\varepsilon_{k}$, respectively, and estimated $\Delta(k)$ which reproduces the experimental band dispersion below $T_{\mathrm{c}}$. The obtained $\Delta(k)$ was strongly $k$-dependent as seen from Figure 4a,b for 1 ML- and Cs-deposited 20 ML-FeSe, respectively; namely, $\Delta(k)$ was finite only in the narrow $k$ region centered at $k_{\mathrm{F}}$ (within $\pm 0.02 \pi / a$ of $k_{\mathrm{F}}$ ), so that band dispersion only around $E_{\mathrm{F}}$ was shifted toward high binding energies by the superconducting transition, consistent with our observations. An unusual Cooper pairing in the limited $k$ space near $k_{\mathrm{F}}$ may be caused by pairing interactions which have small momentum transfer $q$ [25-27]. For instance, it has been proposed by Migdal-Eliashberg theory for 1 ML-FeSe that forward scattering with small $q$ phonons produces highly anisotropic superconducting gap peaked at $k_{\mathrm{F}}$ and also leads to temperature-independent band structure away from $k_{\mathrm{F}}$ [25], in qualitative agreement with $\Delta(k)$ in Figure 4 a as well as band dispersion in Figure 3. Although this theory assumes a cross-interface coupling between small $q$ phonons of $\mathrm{SrTiO}_{3}$ and electrons in 1 ML-FeSe as the key pairing interactions, our observation of anisotropic $\Delta(k)$ in $20 \mathrm{ML}-\mathrm{FeSe}$ (Figure $4 \mathrm{~b}$ ) where interfacial effects are negligible suggests that small- $q$ interactions within the FeSe layers may be also responsible for superconductivity if the $k$-dependent pairing was indeed a source of the deviation from the simulated BQP dispersion.

(a) $1 \mathrm{ML}-\mathrm{FeSe}$

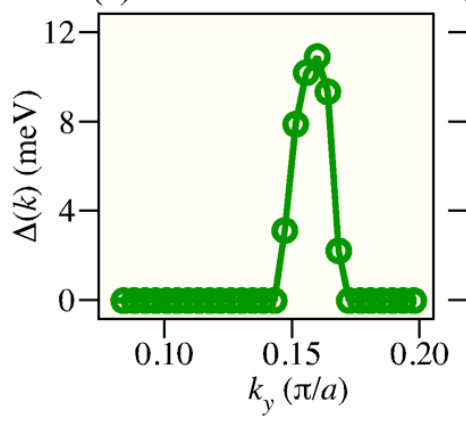

(b) Cs/20ML-FeSe

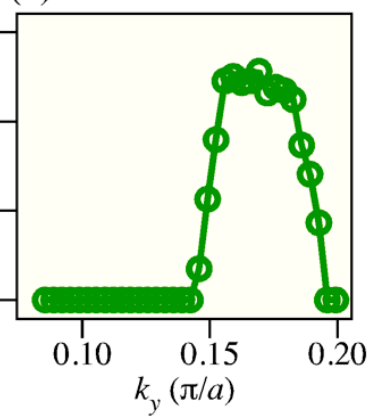

(c) $1 \mathrm{ML}-\mathrm{FeSe}$

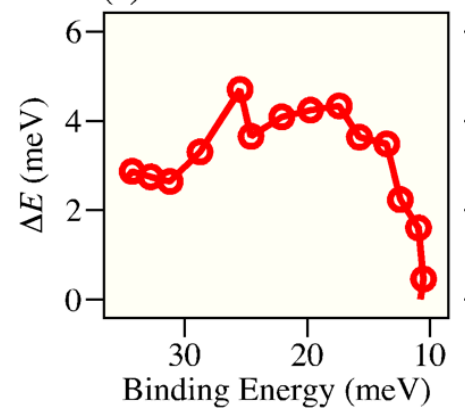

(d) Cs/20ML-FeSe

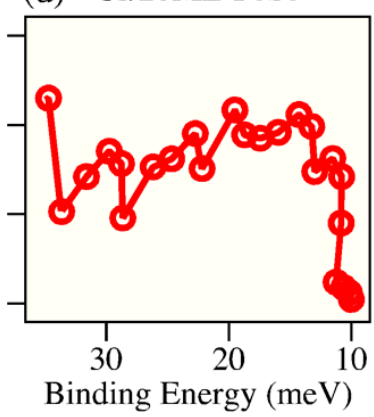

Figure 4. (a) $k$ dependence of the superconducting-gap size $\Delta(k)$ which was calculated to reproduce the experimental band dispersion at $13 \mathrm{~K}$ for $1 \mathrm{ML}-\mathrm{FeSe}$ (blue circles in Figure 3c) with the formula $E_{k}=\sqrt{\varepsilon_{k}^{2}+|\Delta|^{2}}$, where $\varepsilon_{k}$ is the normal-state band dispersion extracted at $T=50 \mathrm{~K}$ (red circles in Figure 3c). (b) Same as (a) but for Cs-deposited 20 ML-FeSe. (c) Energy difference between the experimental band dispersion at $13 \mathrm{~K}$ (blue circles in Figure $3 \mathrm{c}$ ) and the simulated BQP dispersion with a constant gap size of $10 \mathrm{meV}$ (light blue curve in Figure 3c) in $1 \mathrm{ML}-F e S e$. (d) Same as (c) but for Cs-deposited 20 ML-FeSe.

Next, we consider another possibility that $\varepsilon_{k}$ is temperature-dependent whereas $k$ dependence of $\Delta(k)$ is small. To explain the observed deviation between the experimental and simulated $\mathrm{BQP}$ dispersions, $\varepsilon_{k}$ below $T_{\mathrm{c}}$ must be shifted toward $E_{\mathrm{F}}$ compared with the normal-state band dispersion above $T_{\mathrm{C}}$ while keeping the same $k_{\mathrm{F}}$ position. Such an energy shift would be a consequence of mass renormalization linked to the superconducting transition, likely due to coupling with bosonic modes as reported for bulk crystals of high- $T_{\mathrm{c}}$ superconductors [28-33]. Here we defined the energy difference between the experimental and simulated $\mathrm{BQP}$ dispersions as $\Delta E$ (see black arrow in Figure $3 \mathrm{c}$ ) and plotted it in Figure 4c,d for $1 \mathrm{ML}-$ and $20 \mathrm{ML}-F e S e$ films, respectively. As seen from Figure $4 \mathrm{c}, \mathrm{d}, \Delta E$ showed a broad peak around $20 \mathrm{meV}$ in $1 \mathrm{ML}$ - and $20 \mathrm{ML}-\mathrm{FeSe}$. The 
obtained $\Delta E$ value can be used as a measure of the mass enhancement similarly to the real part of self-energies. By analogy with the fact that the peak position in the real part of selfenergies below $T_{\mathrm{c}}$ corresponded to $\Delta+\Omega$, where $\Omega$ is the energy of bosonic modes coupled to electrons, the observed peak structure at $\sim 20 \mathrm{meV}$ suggests a coupling to low-energy modes with $\Omega \sim 10 \mathrm{meV}$ (here, $\Delta \sim 10 \mathrm{meV}$ ). The origin of the corresponding modes is an important open question; candidates include phonons [34] and magnetic resonance [35]. Nevertheless, one important outcome from our observation is that low-energy modes intrinsic to the FeSe layer must be involved because the mass renormalization was found not only in $1 \mathrm{ML}-\mathrm{FeSe}$, but also in $20 \mathrm{ML}-\mathrm{FeSe}$.

In summary, we reported the evolution of low-energy band dispersion across $T_{\mathrm{c}}$ in $1 \mathrm{ML}$ - and Cs-deposited $20 \mathrm{ML}-\mathrm{FeSe}$ films on $\mathrm{SrTiO}_{3}$. We found deviation of the band dispersion below $T_{\mathrm{c}}$ from the simple BQP dispersion simulated with the temperatureindependent $\varepsilon_{k}$ and $k$-independent $\Delta$. We proposed two possible scenarios as the origin of this observation; (i) anisotropic $\Delta(k)$ peaked around $k_{\mathrm{F}}$ due to the superconducting pairing by small $q$ transfer and (ii) enhancement in the effective mass in the superconducting state due to the coupling to low-energy bosonic modes. In either scenario, the observed similarity between $1 \mathrm{ML}$ - and $20 \mathrm{ML}-\mathrm{FeSe}$ suggested intra-FeSe-layer nature of the interactions. Our result lays a foundation for understanding the mechanism of high- $T_{\mathrm{c}}$ superconductivity in electron-doped FeSe.

Author Contributions: Conceptualization, K.N.; formal analysis, K.S. (Koshin Shigekawa); investigation, K.N., K.S. (Koshin Shigekawa) and K.S. (Katsuaki Sugawara); data curation, K.N. and K.S. (Koshin Shigekawa); writing—original draft preparation, K.N.; writing—review and editing, T.T. and T.S.; visualization, K.N. and K.S. (Koshin Shigekawa); project administration, K.N. and T.S.; funding acquisition, K.N., K.S. (Katsuaki Sugawara), T.T. and T.S. All authors have read and agreed to the published version of the manuscript.

Funding: This work was supported by JST-CREST (No. JPMJCR18T1) and Grant-in-Aid for Scientific Research (JSPS KAKENHI Grant Numbers JP17H04847, JP17H01139, JP18H01160, and JP20H01847).

Institutional Review Board Statement: Not applicable.

Informed Consent Statement: Not applicable.

Data Availability Statement: The data that support the findings of this study are available from the corresponding author upon request.

Acknowledgments: We thank G. Phan and M. Kuno for their assistance in the ARPES measurements.

Conflicts of Interest: The authors declare no conflict of interests.

\section{References}

1. Hsu, F.C.; Luo, J.-Y.; Yeh, K.-W.; Chen, T.-K.; Huang, T.-W.; Wu, P.M.; Lee, Y.-C.; Huang, Y.-L.; Chu, Y.-Y.; Yan, D.-C.; et al. Superconductivity in the PbO-type structure $\alpha$-FeSe. Proc. Natl. Acad. Sci. USA 2009, 105, 14262-14264. [CrossRef] [PubMed]

2. Wang, Q.Y.; Wang, Q.-Y.; Li, Z.; Zhang, W.-H.; Zhang, Z.-C.; Zhang, J.-S.; Li, W.; Ding, H.; Ou, Y.-B.; Deng, P.; et al. Interfaceinduced high-temperature superconductivity in single unit-cell FeSe films on $\mathrm{SrTiO}_{3}$. Chin. Phys. Lett. $2012,29,037402$. [CrossRef]

3. Sun, Y.; Zhang, W.; Xing, Y.; Li, F.; Zhao, Y.; Xia, Z.; Wang, L.; Ma, X.; Xue, Q.-K.; Wang, J. High temperature superconducting FeSe films on $\mathrm{SrTiO}_{3}$ substrates. Sci. Rep. 2014, 4, 6040. [CrossRef] [PubMed]

4. He, S.L.; He, S.; Zhang, W.; Zhao, L.; Liu, D.; Liu, X.; Mou, D.; Ou, Y.-B.; Wang, Q.-Y.; Li, Z.; et al. Phase diagram and electronic indication of high-temperature superconductivity at $65 \mathrm{~K}$ in single-layer FeSe films. Nat. Mater. 2013, 12, 605-610. [CrossRef]

5. Tan, S.Y.; Zhang, Y.; Xia, M.; Ye, Z.; Chen, F.; Xie, X.; Peng, R.; Xu, D.; Fan, Q.; Xu, H.; et al. Interface-induced superconductivity and strain-dependent spin density wave in $\mathrm{FeSe} / \mathrm{SrTiO}_{3}$ thin films. Nat. Mater. 2013, 12, 634-640. [CrossRef]

6. Peng, R.; Xu, H.C.; Tan, S.Y.; Cao, H.Y.; Xia, M.; Shen, X.P.; Huang, Z.C.; Wen, C.H.P.; Song, Q.; Zhang, T.; et al. Tuning the band structure and superconductivity in single-layer FeSe by interface engineering. Nat. Commun. 2014, 5, 5044. [CrossRef]

7. Peng, R.; Shen, X.P.; Xie, X.; Xu, H.C.; Tan, S.Y.; Xia, M.; Zhang, T.; Cao, H.Y.; Gong, X.G.; Hu, J.P.; et al. Measurement of an Enhanced Superconducting Phase and a Pronounced Anisotropy of the Energy Gap of a Strained FeSe Single Layer in $\mathrm{FeSe} / \mathrm{Nb}: \mathrm{SrTiO}_{3} / \mathrm{KTaO}_{3}$ Heterostructures Using Photoemission Spectroscopy. Phys. Rev. Lett. 2014, 112, 107001. [CrossRef] 
8. Zhang, Z.; Wang, Y.-H.; Song, Q.; Liu, C.; Peng, R.; Moler, K.A.; Feng, D.; Wang, Y. Onset of the Meissner effect at 65 K in FeSe thin film grown on $\mathrm{Nb}$-doped $\mathrm{SrTiO}_{3}$ substrate. Sci. Bull. 2015, 60, 1301-1304. [CrossRef]

9. Lee, J.J.; Schmitt, F.T.; Moore, R.G.; Johnston, S.; Cui, Y.-T.; Li, W.; Yi, M.; Liu, Z.K.; Hashimoto, M.; Zhang, Y.; et al. Interfacial mode coupling as the origin of the enhancement of $T_{\mathrm{c}}$ in FeSe films on $\mathrm{SrTiO}_{3}$. Nature 2014, 515, 245. [CrossRef]

10. Xiang, Y.Y.; Wang, F.; Wang, D.; Wang, Q.H.; Lee, D.H. High-temperature superconductivity at the FeSe $/ \mathrm{SrTiO}_{3}$ interface. Phys. Rev. B 2012, 86, 134508. [CrossRef]

11. Song, Q.; Yu, T.L.; Lou, X.; Xie, B.P.; Xu, H.C.; Wen, C.H.P.; Yao, Q.; Zhang, S.Y.; Zhu, X.T.; Guo, J.D.; et al. Evidence of cooperative effect on the enhanced superconducting transition temperature at the FeSe/SrTiO 3 interface. Nat. Commun. 2019, 10, 758. [CrossRef] [PubMed]

12. Rebec, S.N.; Jia, T.; Zhang, C.; Hashimoto, M.; Lu, D.-H.; Moore, R.G.; Shen, Z.-X. Coexistence of Replica Bands and Superconductivity in FeSe Monolayer Films. Phys. Rev. Lett. 2017, 118, 067002. [CrossRef] [PubMed]

13. Li, F.; Ding, H.; Tang, C.; Peng, J.; Zhang, Q.; Zhang, W.; Zhou, G.; Zhang, D.; Song, C.-L.; He, K.; et al. Interface-enhanced high-temperature superconductivity in single-unit-cell FeTe ${ }_{1-x} \mathrm{Se}_{x}$ films on $\mathrm{SrTiO}_{3}$. Phys. Rev. B 2015, 91, 220503. [CrossRef]

14. Shi, X.; Han, Z.-Q.; Richard, P.; Wu, X.-X.; Peng, X.-L.; Qian, T.; Wang, S.-C.; Hu, J.-P.; Sun, Y.-J.; Ding, H. FeTe ${ }_{1-x}$ Se $_{x}$ monolayer films: Towards the realization of high-temperature connate topological superconductivity. Sci. Bull. 2017, 62, 503-507. [CrossRef]

15. Shigekawa, K.; Nakayama, K.; Kuno, M.; Phan, G.N.; Owada, K.; Sugawara, K.; Takahashi, T.; Sato, T. Dichotomy of superconductivity between monolayer FeS and FeSe. Proc. Natl. Acad. Sci. USA 2019, 116, 24470-24474. [CrossRef]

16. Liu, D.F.; Zhang, W.; Mou, D.; He, J.; Ou, Y.-B.; Wang, Q.-Y.; Li, Z.; Wang, L.; Zhao, L.; He, S.; et al. Electronic origin of high-temperature superconductivity in single-layer FeSe superconductor. Nat. Commun. 2012, 3, 931. [CrossRef]

17. Maletz, J.; Zabolotnyy, V.B.; Evtushinsky, D.V.; Thirupathaiah, S.; Wolter, A.U.B.; Harnagea, L.; Yaresko, A.N.; Vasiliev, A.N.; Chareev, D.A.; Böhmer, A.E.; et al. Unusual band renormalization in the simplest iron-based superconductor FeSe $1-x$. Phys. Rev. B 2014, 89, 220506. [CrossRef]

18. Nakayama, K.; Miyata, Y.; Phan, G.N.; Sato, T.; Tanabe, Y.; Urata, T.; Tanigaki, K.; Takahashi, T. Reconstruction of Band Structure Induced by Electronic Nematicity in an FeSe Superconductor. Phys. Rev. Lett. 2014, 113, 237001. [CrossRef]

19. Miyata, Y.; Nakayama, K.; Sugawara, K.; Sato, T.; Takahashi, T. High-temperature superconductivity in potassium-coated multilayer FeSe thin films. Nat. Mater. 2015, 14, 775-779. [CrossRef]

20. Wen, C.H.P.; Xu, H.C.; Chen, C.; Huang, Z.C.; Lou, X.; Pu, Y.J.; Song, Q.; Xie, B.P.; Abdel-Hafiez, M.; Chareev, D.A.; et al. Anomalous correlation effects and unique phase diagram of electron-doped FeSe revealed by photoemission spectroscopy. Nat. Commun. 2016, 7, 10840. [CrossRef]

21. Shiogai, J.; Ito, Y.; Mitsuhashi, T.; Nojima, T.; Tsukazaki, A. Electric-field-induced superconductivity in electrochemically etched ultrathin FeSe films on $\mathrm{SrTiO}_{3}$ and MgO. Nat. Phys. 2016, 12, 42-46. [CrossRef]

22. Phan, G.N.; Nakayama, K.; Kanayama, S.; Kuno, M.; Sugawara, K.; Sato, T.; Takahashi, T. ARPES study of cesium-coated FeSe thin films on $\mathrm{SrTiO}_{3}$. J. Phys. Conf. Ser. 2017, 871, 012017. [CrossRef]

23. Norman, M.R.; Randeria, M.; Ding, H.; Campuzano, J.C. Phenomenology of the low-energy spectral function in high- $T_{\mathrm{c}}$ superconductors. Phys. Rev. B 1998, 57, R11093-R11096. [CrossRef]

24. Matsui, H.; Sato, T.; Takahashi, T.; Wang, S.-C.; Yang, H.-B.; Ding, H.; Fujii, T.; Watanabe, T.; Matsuda, A. BCS-Like Bogoliubov Quasiparticles in High- $T_{\mathrm{c}}$ Superconductors Observed by Angle-Resolved Photoemission Spectroscopy. Phys. Rev. Lett. 2003, 90, 217002. [CrossRef] [PubMed]

25. Wang, Y.; Nakatsukasa, K.; Rademaker, L.; Berlijn, T.; Johnston, S. Aspects of electron-phonon interactions with strong forward scattering in FeSe Thin Films on $\mathrm{SrTiO}_{3}$ substrates. Supercond. Sci. Technol. 2016, 29, 054009. [CrossRef]

26. Yamase, H.; Zeyher, R. Superconductivity from orbital nematic fluctuations. Phys. Rev. B 2013, 88, 180502. [CrossRef]

27. Agatsuma, T.; Yamase, H. Structure of the pairing gap from orbital nematic fluctuations. Phys. Rev. B 2016, 94, 214505. [CrossRef]

28. Norman, M.R.; Ding, H.; Campuzano, J.C.; Takeuchi, T.; Randeria, M.; Yokoya, T.; Takahashi, T.; Mochiku, T.; Kadowaki, K. Unusual Dispersion and Line Shape of the Superconducting State Spectra of $\mathrm{Bi}_{2} \mathrm{Sr}_{2} \mathrm{CaCu}_{2} \mathrm{O}_{8+\delta}$. Phys. Rev. Lett. 1997, 79, 3506-3509. [CrossRef]

29. Valla, T.; Fedorov, A.V.; Johnson, P.D.; Wells, B.O.; Hulbert, S.L.; Li, Q.; Gu, G.D.; Koshizuka, N. Evidence for Quantum Critical Behavior in the Optimally Doped Cuprate $\mathrm{Bi}_{2} \mathrm{Sr}_{2} \mathrm{CaCu}_{2} \mathrm{O}_{8+\delta}$. Science 1999, 285, 2110. [CrossRef]

30. Kaminski, A.; Randeria, M.; Campuzano, J.C.; Norman, M.R.; Fretwell, H.; Mesot, J.; Sato, T.; Takahashi, T.; Kadowaki, K. Renormalization of Spectral Line Shape and Dispersion below $T_{\mathrm{c}}$ in $\mathrm{Bi}_{2} \mathrm{Sr}_{2} \mathrm{CaCu}_{2} \mathrm{O}_{8+\delta}$. Phys. Rev. Lett. 2001, 86, 1070. [CrossRef]

31. Lanzara, A.; Bogdanov, P.V.; Zhou, X.J.; Kellar, S.A.; Feng, D.L.; Lu, E.D.; Yoshida, T.; Eisaki, H.; Fujimori, A.; Kishio, K.; et al. Evidence for ubiquitous strong electron-phonon coupling in high-temperature superconductors. Nature 2001, 412, 510. [CrossRef] [PubMed]

32. Richard, P.; Sato, T.; Nakayama, K.; Souma, S.; Takahashi, T.; Xu, Y.-M.; Chen, G.F.; Luo, J.L.; Wang, N.L.; Ding, H. Angle-Resolved Photoemission Spectroscopy of the Fe-Based $\mathrm{Ba}_{0.6} \mathrm{~K}_{0.4} \mathrm{Fe}_{2} \mathrm{As}_{2}$ High Temperature Superconductor: Evidence for an Orbital Selective Electron-Mode Coupling. Phys. Rev. Lett. 2009, 102, 047003. [CrossRef] [PubMed]

33. Koitzsch, A.; Inosov, D.S.; Evtushinsky, D.V.; Zabolotnyy, V.B.; Kordyuk, A.A.; Kondrat, A.; Hess, C.; Knupfer, M.; Büchner, B.; Sun, G.L.; et al. Temperature and Doping-Dependent Renormalization Effects of the Low Energy Electronic Structure of $\mathrm{Ba}_{1-x} \mathrm{~K}_{x} \mathrm{Fe}_{2} \mathrm{As}_{2}$ Single Crystals. Phys. Rev. Lett. 2009, 102, 167001. [CrossRef] [PubMed] 
34. Tang, C.; Liu, C.; Zhou, G.; Li, F.; Ding, H.; Li, Z.; Zhang, D.; Li, Z.; Song, C.; Ji, S.; et al. Interface-enhanced electron-phonon coupling and high-temperature superconductivity in potassium-coated ultrathin FeSe films on $\mathrm{SrTiO}_{3}$. Phys. Rev. B 2016, 93, 020507. [CrossRef]

35. Ma, M.; Bourges, P.; Sidis, Y.; Xu, Y.; Li, S.; Hu, B.; Li, J.; Wang, F.; Li, Y. Prominent Role of Spin-Orbit Coupling in FeSe Revealed by Inelastic Neutron Scattering. Phys. Rev. X 2017, 7, 021025. [CrossRef] 